\title{
Moxibustion for cancer-related fatigue: study protocol for a randomized controlled trial
}

Mikyung Kim¹, Jung-Eun Kim¹, Hye-Yoon Lee', Ae-Ran Kim¹, Hyo-Ju Park', O-Jin Kwon', Eun-Jung Kim², Yeon-Cheol Park', Byung-Kwan Seo ${ }^{3}$, Jung Hyo $\mathrm{Cho}^{4}$ and Joo-Hee Kim ${ }^{1 *}$

\begin{abstract}
Background: Cancer-related fatigue is one of the most common symptoms experienced by cancer patients, and it diminishes their quality of life. However, there is currently no confirmed standard treatment for cancer-related fatigue, and thus, many patients who suffer cancer-related fatigue seek complementary and alternative medicines such as moxibustion. Moxibustion is one of the most popular therapies in traditional Korean medicine used to manage fatigue. Recent studies have also demonstrated that moxibustion is effective for treating chronic fatigue. However, there is insufficient evidence supporting the effect of moxibustion against cancer-related fatigue. The aim of this study is to assess the efficacy and safety of moxibustion treatment for cancer-related fatigue.

Methods/design: A multi-center, three-armed parallel, randomized controlled trial will be conducted. Ninety-six patients with cancer-related fatigue will be recruited from three clinical research centers. They will be randomly allocated to one of three groups in a 1:1:1 ratio. The moxibustion group will receive moxibustion treatment at CV8, CV12, LI4 and ST36. The sham moxibustion group will receive sham moxibustion at non-acupoints. Both the moxibustion and sham moxibustion groups will receive 30-min treatments twice a week for 8 weeks. The usual care group will not receive moxibustion treatment. All participants will be educated via a brochure on how to manage cancer-related fatigue in daily life. The outcome measurements will be evaluated at baseline, week 5, week 9, and week 13 by assessors who are blinded to the group allocation. The primary outcome measure will be the mean change in the average scores of the Brief Fatigue Inventory before and after treatments between groups. The secondary outcome measures will be the mean difference in changes from baseline of the Brief Fatigue Inventory, functional assessments of cancer therapy-fatigue, European Organization for Research and Treatment of Cancer Quality of Life Questionnaire C-30 scores, and Montreal Cognitive Assessment scores between groups. Safety will be assessed by monitoring adverse events at each visit.
\end{abstract}

Discussion: The results of this study will provide evidence to confirm whether moxibustion can be used as a therapeutic option for treating cancer-related fatigue.

Trial registration: Clinical Research Information Service KCT0002170. Registered 16 December 2016.

Keywords: Cancer-related fatigue, Moxibustion, Randomized controlled trial, Traditional Korean medicine

\footnotetext{
* Correspondence: jhkim714v@gmail.com

${ }^{1}$ Clinical Research Division, Korea Institute of Oriental Medicine, 1672,

Yuseongdae-ro, Yuseong-gu, Daejeon 34054, Republic of Korea

Full list of author information is available at the end of the article
}

(c) The Author(s). 2017 Open Access This article is distributed under the terms of the Creative Commons Attribution 4.0 International License (http://creativecommons.org/licenses/by/4.0/), which permits unrestricted use, distribution, and reproduction in any medium, provided you give appropriate credit to the original author(s) and the source, provide a link to the Creative Commons license, and indicate if changes were made. The Creative Commons Public Domain Dedication waiver (http://creativecommons.org/publicdomain/zero/1.0/) applies to the data made available in this article, unless otherwise stated. 


\section{Background}

As the prevalence of cancer and the survival rate of patients increase, the quality of life (QOL) of cancer survivors and the symptoms affecting $\mathrm{QOL}$ are gaining increased attention [1]. Cancer-related fatigue is one of the most common symptoms that cancer patients experience [2] and is among the most prominent residual or persistent symptoms of cancer survivors [3]. Cancerrelated fatigue often occurs during the cancer treatment period, although it may occur independent of treatment and sometimes continues to persist even after the end of treatment or the complete remission of cancer [4].

Cancer-related fatigue reduces the level of energy and decreases the QOL of cancer patients, negatively affecting the return-to-work rate of cancer survivors $[5,6]$. It also seriously damages the QOL of the families in addition to cancer patients in terms of physical, social, economic, psychological and spiritual aspects [7]. However, it is not a fatal condition that directly threatens the survival of cancer patients. Therefore, it is recognized to be relatively less important than other critical issues regarding cancer [8]. Moreover, the definition of cancerrelated fatigue is completely dependent on the subjective sense of tiredness felt by the patient, and therefore, it is easy to overlook when the patient does not actively report the symptom [8].

The mechanism of cancer-related fatigue has been reported to be associated with cytokine production or mediators of inflammatory responses of cancer and/or cancer treatment [9]. In particular, tumor necrosis factor (TNF) alpha signaling seems to be closely related to cancer-related fatigue [6]. In addition to inflammation and latent viral reactivation $[10,11]$, other various factors such as oxidative stress [12], changes in the neuroendocrine system including dysregulation of the hypothalamic-pituitary-adrenal (HPA) axis, autonomic nervous system disorders including central vagal stimulation, serotonin dysregulation [10,11], and changes in muscle and ATP metabolism [11], have been reported to be involved in cancer-related fatigue. However, the mechanism of cancer-related fatigue has not yet been fully elucidated [13].

There are various known risk factors related to cancer-related fatigue, including the stage of cancer, the specific type of cancer (breast, colon, liver) and diabetes, high body mass index, genetic factor, psychological and biobehavioral factors such as the level of fatigue before cancer treatment, depression, sleep disturbances, physical activity and physical deconditioning, negative expectation, etc. $[10,14,15]$. The cancer itself, various cancer treatments, and accompanying diseases such as anemia, cachexia, etc., also appear to be related to cancer-related fatigue, but the degree of their contributions and how such risk factors interact remain unclear
[11]. To date, the cause of cancer-related fatigue has not been explicitly identified [11, 14].

The first step in managing cancer-related fatigue is determining the possible causes of fatigue and correcting them $[4,8,16]$. However, as previously discussed, a variety of factors are involved and contribute to cancerrelated fatigue, and therefore, it is very difficult to identify specific causes and to completely eliminate fatigue among cancer patients [14]. There is currently no standard therapy for treating cancer-related fatigue [6, 17].

For pharmacologic therapy to mitigate cancer-related fatigue, antidepressants and psychostimulants such as methylphenidate, modafinil, and bupropion and steroids such as dexamethasone have been proposed and studied, but the results of clinical trials are inconsistent $[4,16-18]$. According to a recent meta-analysis, methylphenidate was significantly better than placebo, while the effect of modafinil was similar to that of placebo, although further studies are needed to confirm this conclusion [17]. Considering that the clinical effects of these drugs are unclear and the risk of adverse drug reactions are of high concern, especially for long-term use, no specific drug is currently recommended as a primary therapeutic option for cancerrelated fatigue $[17,19,20]$.

Despite the lack of a standard therapy for cancerrelated fatigue, the high number of patients who suffer from cancer-related fatigue has created a demand for complementary alternative medicine (CAM). A systematic review reported that CAM utilization rates among cancer patients range from 11 to 95\% [21]. According to a survey targeting breast cancer survivors in the United States, one third of patients used CAM [22]. Another survey conducted among South Korean cancer patients reported that more than half of respondents used CAM [23].

Moxibustion is one of the most commonly used treatments in traditional medicine in East Asia including Korea. It has been used to prevent and treat various diseases by warming the body and promoting the energy of patients [24, 25]. Moxibustion is particularly good at supplementing energy $(q i)$, and thus, it has been often applied to patients who experience fatigue or similar conditions [25]. Modern scientific studies also have shown that moxibustion is effective in alleviating chronic fatigue [26-28], and some parts of its mechanism have been reported to be related to activation of the vagus nerve [26], reduction of oxidative damage [26, 27, 29], improvement of inflammation [30], regulation of the HPA axis [10], and upregulation of hippocampal progranulin [10].

Lee et al. [31] showed the possibility of using moxibustion to treat cancer-related fatigue through a systematic review and meta-analysis of related literature. However, all the studies included in this review were reported and 
conducted only in China, and most of the studies had high risk of bias. The details of moxibustion treatment used in each study were not described based on the Consolidated Standards of Reporting Trials (CONSORT) or on the Standards for Reporting Interventions in Clinical Trials of Acupuncture (STRICTA), and most of the studies did not report of adverse events (AEs) and dropouts. Thus, Lee et al. [31] concluded that a welldesigned clinical trial with better reporting quality is warranted to draw any conclusion regarding the effect of moxibustion for treatment of cancer-related fatigue. Recently, Mao et al. [32] demonstrated that their newly devised method of infrared laser moxibustion was more effective in reducing the level of fatigue than sham moxibustion. However, infrared laser moxibustion is not yet commonly used in Korea. More evidence is needed to confirm the effectiveness and safety of moxibustion against cancer-related fatigue.

Therefore, we designed this randomized controlled trial to compare the clinical responses of moxibustion, sham moxibustion, and usual care interventions in patients with cancer-related fatigue.

\section{Methods/design}

\section{Design}

This is a multi-center, three-armed parallel, randomized controlled trial. Ninety-six participants who meet the diagnostic criteria for cancer-related fatigue of the international classification of diseases, tenth revision clinical modification (ICD-10-CM), will be randomly allocated to one of the three groups in a 1:1:1 ratio. One group will be the treatment group (moxibustion group), and the other two will be control groups (sham moxibustion group and usual care group). The flowchart of this trial is shown in Fig. 1.

\section{Recruitment}

Three clinical research centers in South Korea will recruit participants: Daejeon Oriental Hospital of Daejeon University, Kyung Hee University Oriental Hospital at Gangdong, and Dongguk University Bundang Oriental Hospital.

To ensure enough patients, clinical trial information will be posted on notice boards of the hospitals both on and offline. Recruitment notices will also be released on flyers, daily local newspapers, and advertisement boards at public places.

\section{Eligibility criteria Inclusion criteria}

1. Male or female aged 19 or over but under 80 years

2. Cessation of cancer-related treatments (e.g., surgery, chemotherapy, radiotherapy, immunotherapy) at

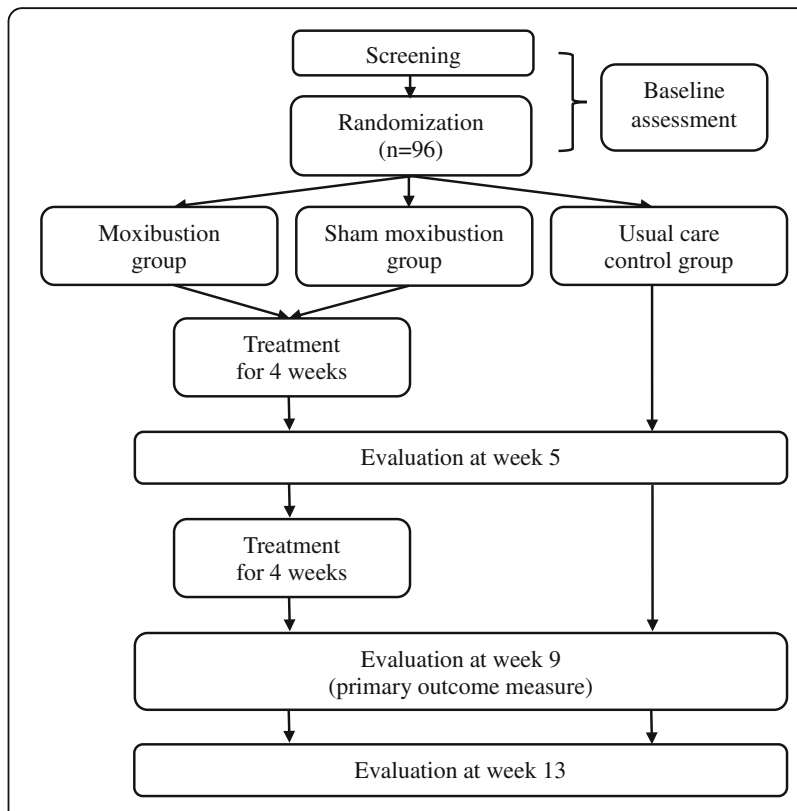

Fig. 1 Flow chart of the study

least 12 weeks before the trial (on-going hormone therapy, which must have been initiated at least 3 weeks prior to enrollment, is allowed)

3. Continuous fatigue related to cancer treatment or cancer itself for at least 4 weeks, fulfilment of the ICD-10-CM diagnostic criteria for cancer-related fatigue, and a brief fatigue inventory (BFI) score of 4 or more

4. An Eastern Cooperative Oncology Group (ECOG) performance status $\leq 2$

5. Willingness to participate in the trial and having provided written consent

\section{Exclusion criteria}

1. Having maintained the current level of fatigue prior to the diagnosis of cancer

2. Severe anemia (platelet count $<60,000 / \mu \mathrm{L}$, hemoglobin $<8 \mathrm{~g} / \mathrm{dL}$, or absolute neutrophil count $<1000 / \mu \mathrm{L}$ )

3. Receiving aggressive treatment for anemia (e.g., erythropoietin or blood transfusion)

4. Poor oral intake with a lower-than-normal level of serum albumin

5. Any significant sign or symptom of inflammation with C-reactive protein $(C R P) \geq 10 \mathrm{mg} / \mathrm{L}$ and white blood cell $>10,000 / \mu \mathrm{L}$

6. Abnormal findings in a thyroid function test (abnormal level of free thyroxine and thyroid stimulating hormone $<0.1 \mu \mathrm{IU} / \mathrm{ml}$ or TSH $>5.1 \mu \mathrm{IU} / \mathrm{ml}$ ) 
7. Abnormal findings in a liver function test or a renal function test (aspartate aminotransferase or alanine aminotransferase $\geq 2 \times$ upper normal range or creatinine $\geq 2.0 \mathrm{mg} / \mathrm{dL}$ ) or the presence of serious liver failure or renal failure

8. A score of 11 points or more on either the anxiety or depression subscales of the Hospital Anxiety and Depression Scale (HADS)

9. An insomnia severity index (ISI) score of 15 points or more

10. Level of cancer pain measured by the numeric rating scale (NRS) $\geq 4$

11. An estimated life expectancy of six months or less

12. A plan of surgery, chemotherapy, or radiotherapy during the study

13. History of medication (methylphenidate, modafinil, bupropion, dexamethasone) to manage cancerrelated fatigue taken within 4 weeks of the beginning of the trial

14. History of Korean medical treatment (e.g., acupuncture, moxibustion, cupping, or herbal medicine, etc.) to manage cancer-related fatigue within 4 weeks of the beginning of the trial

15. Initiation or a change in dietary supplement regimen or non-pharmacologic therapies (e.g., cognitive behavioral therapy, exercise, etc.) for alleviating cancer-related fatigue during the trial or within 4 weeks of the beginning of the trial

16. Having participated in other clinical trials within 4 weeks of the beginning of the trial

17. History of hypersensitivity reactions or serious adverse reactions (SAEs) after moxibustion treatment or an inability to cooperate with moxibustion treatment due to other reasons such as dyspnea

18. Women who are pregnant, lactating, or planning to become pregnant

19. Presence of other apparent factors or any diseases that could cause current fatigue other than cancer treatment or the cancer itself

\section{Randomization and allocation concealment}

Random numbers will be generated by SAS (Version 9.4, SAS institute. Inc., Cary, NC) and sealed in sequentially numbered opaque envelopes. The envelopes will be delivered to each research center and kept in a doublelocked cabinet. The practitioners of each research center will open the envelops and allocate the participants who meet the eligibility criteria after signing an informed consent to one of the three groups according to the contents of the envelops.

\section{Blinding}

Practitioners cannot be blinded because of the unique nature of moxibustion treatment. Although the subjects of the usual care group will be able to determine which group they are allocated to, the participants in the moxibustion and sham moxibustion groups will be blinded. To assess whether the patient-blinding was successfully achieved among the moxibustion group and sham moxibustion group, the subjects of these two groups will undergo a blinding test and a credibility test after the first and final treatment. Outcomes will be assessed by researchers who are not involved in the intervention procedure. Thus, the assessors will be blinded to all three groups, while the participants will be blinded in only the moxibustion and sham moxibustion groups.

\section{Intervention}

There are three arms in this trial. All participants regardless of their assignment will be educated via a brochure on how to manage daily life to alleviate the cancer-related fatigue.

\section{Moxibustion group}

Participants in the moxibustion group will receive moxibustion treatment via two types of moxibustion devices: an ignition-type moxibustion device (IM, Fig. 2) and an electrical moxibustion apparatus (EM, Fig. 3).

The IM is composed of a red clay body covered by a silicon sheath and safety cap (Fig. 2). An ignited mugwort moxa cone will be inserted into the body of the IM. The base of the body has tiny holes that acts as a heat channel. Two IMs will be sequentially applied at two acupoints on the abdomen, CV8 and CV12 (Fig. 4).

The EM is a cube-shaped apparatus with a base embedded in an electrical board controlled by a heat sensor (Fig. 3). The EM can be stably fixed on the body by attaching double-sided medical tape to the base of the EM. Four EMs will be sequentially attached at acupoints on the hands and legs, bilateral LI4 and ST36 (Fig. 4). When the button on the top of the EM is pushed, the board on the base starts to emit heat and the light on the top begins to blink. The plateau temperature of the base of the EM is $45{ }^{\circ} \mathrm{C}$. The treatment time is $30 \mathrm{~min}$, and the participants in this group will receive the treatment twice per week for 8 weeks. Detailed explanations of the moxibustion treatments are tabulated according to the revised STRICTA checklist for moxibustion (Additional file 1).

\section{Sham moxibustion group}

The appearances of the IM and EM used for the sham moxibustion group will be exactly identical to those used for the moxibustion group (Fig. 5). The participants will be able to see the practitioner ignite the mugwort and the IM with a small amount of smoke and burning odor applied on their abdomen. In the case of the EM, the participants will be able to see that the EM attached on 


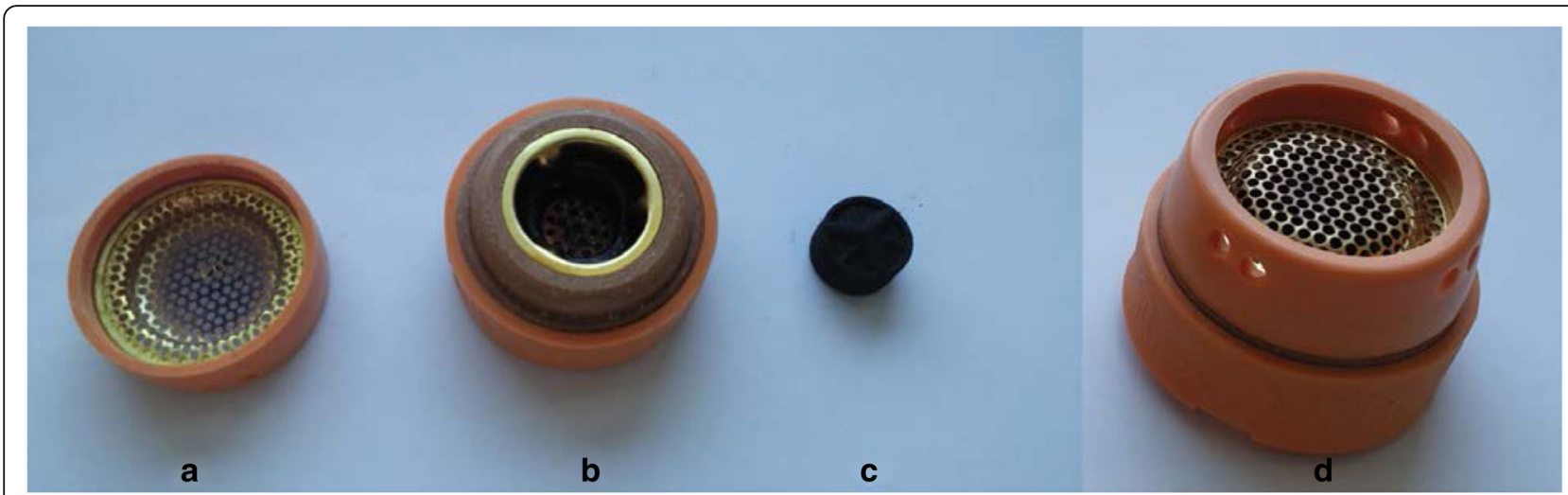

Fig. $\mathbf{2}$ Ignition-type moxibustion device. a, safety cap; $\mathbf{b}$, body of the device; $\mathbf{c}$, mugwort moxa cone; $\mathbf{d}$, the assembled device of $\mathbf{a}$, $\mathbf{b}$, $\mathbf{c}$ and $\mathbf{d}$

their skin is turned on as the light on the top of the EM begins to blink. These procedures will be the same as the method used for the moxibustion group.

However, the base of the IM will be filled with insulator made of styrofoam, and the heat sensor in the electrical board of the base of the EM will be controlled to block heat transfer (Fig. 6). The sham moxibustion will be applied at non-acupoints that are irrelevant to fatigue. The locations of the points that will be used for this group are as follows (Fig. 7): abdomen (approximately $13.5 \mathrm{~cm}$ above the bilateral $3 \mathrm{~cm}$ from the umbilicus), upper limb $(1 \mathrm{~cm}$ lateral and $5 \mathrm{~cm}$ distal to the cubital creases of the bilateral arms), and lower limb (upper $1 / 3$ points of the medial line of the bilateral tibia). The sham
IMs will be applied on the abdomen points, and the sham EMs will be attached on the upper and lower limb points.

\section{Usual care group}

The participants in the usual care group will not receive moxibustion treatment. They will maintain their usual treatment and self-care but will not begin any additional treatment to improve their cancer-related fatigue during the study.

\section{Prohibited and permitted concomitant treatment}

The participants will be prohibited from receiving any pharmacologic treatment of Western medicine or any

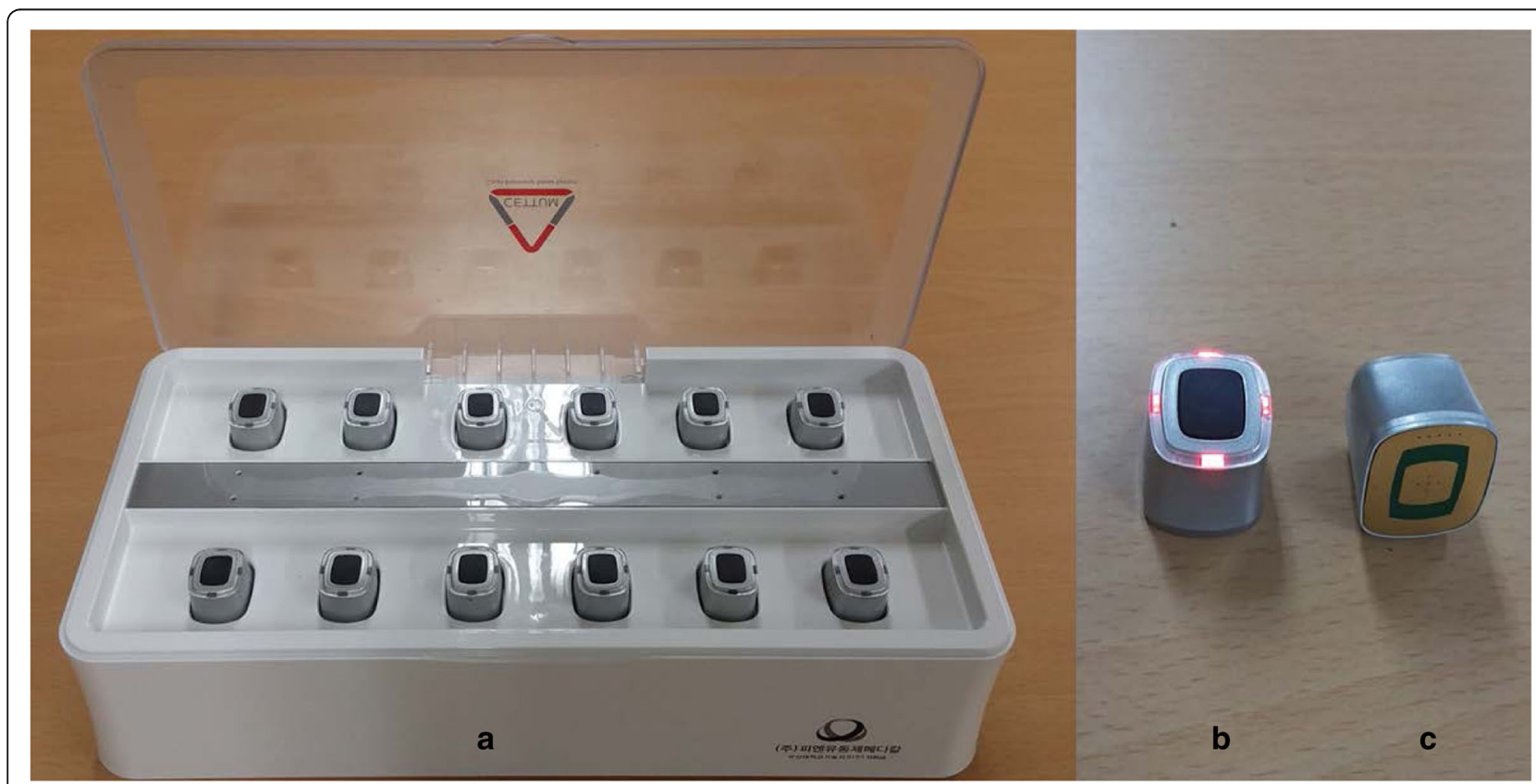

Fig. 3 Electrical moxibustion apparatus. a, twelve moxibustion devices in a charger; $\mathbf{b}$, a powered-up moxibustion device; $\mathbf{c}$ : the bottom of the moxibustion device embedded with an electrically heating board 

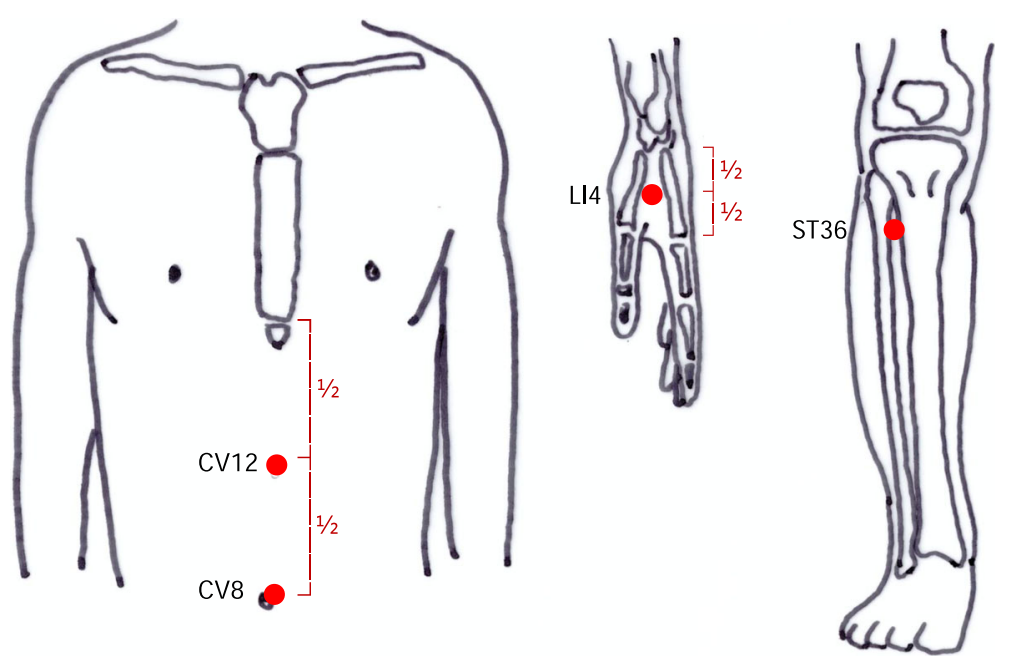

Fig. 4 Acupoints for the moxibustion group

other interventions of traditional Korean medicine to manage cancer-related fatigue other than the interventions provided by the trial. If they continued to receive other nonpharmacologic therapies such as exercise, yoga, meditation, etc. or were already taking any dietary supplements to mitigate cancer-related fatigue at least 4 weeks before the trial was initiated, they will be able to maintain the therapies. However, any treatment used to attenuate cancer-related fatigue cannot be newly introduced after the initiation of the trial. All new treatments begun after the beginning of the trial and concomitant medications to treat medical conditions unrelated to cancer-related fatigue will be recorded on the case report form.
Study schedule of enrolment, allocation, and assessments After eligibility screening of the candidate participants who had signed informed consent, the participants will be randomly allocated to one of the three groups. Before the initial intervention begins, baseline measures will be obtained. After four weeks of intervention, outcomes reflecting the level of fatigue will be assessed at week 5. Post-treatment assessments will be conducted at week 9 after completing eight weeks of intervention. The follow-up visit will be planned at week 13, concluding the trial. The schedule of the enrolment, interventions, and assessments is given in Table 1.

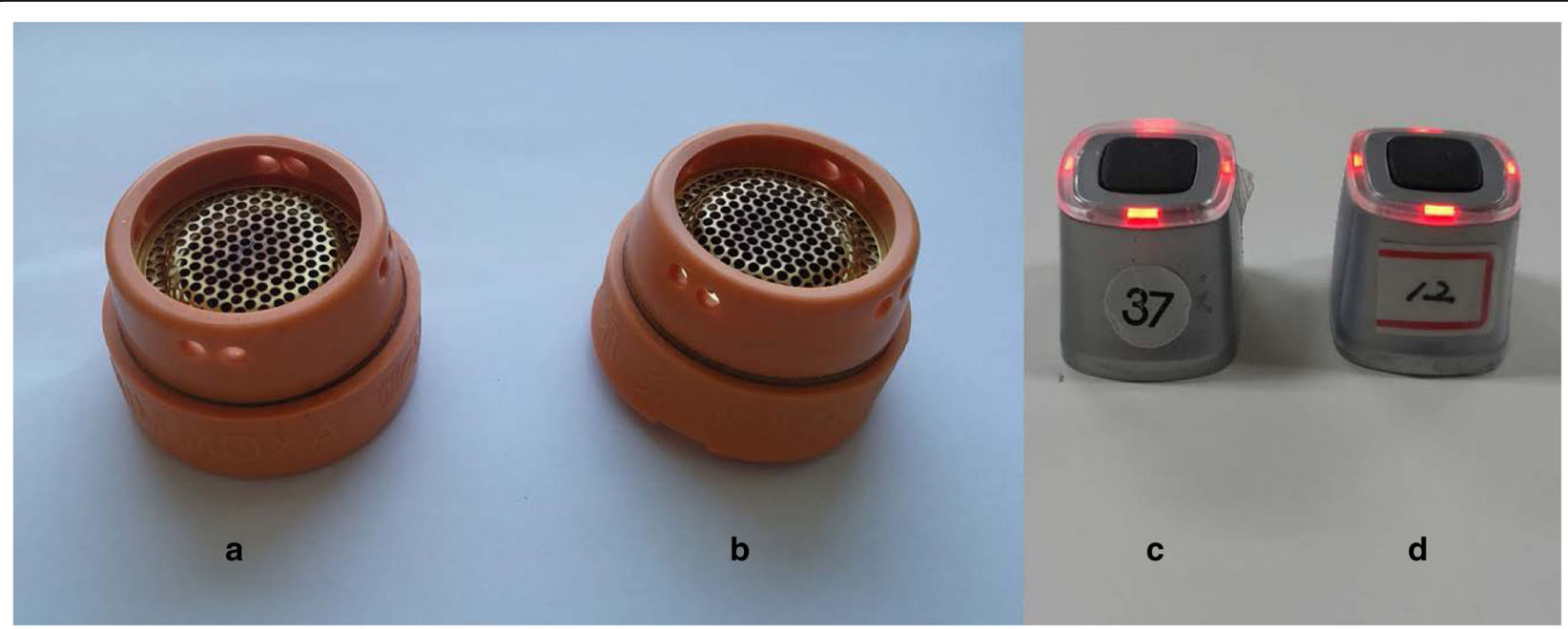

Fig. 5 A front view of moxibustion. a \& b, ignition-type moxibustion devices; $\mathbf{a}$, real; b, sham; c \& d: electrical moxibustion apparatus; c, real; d, sham. The front views of the real and sham moxibustion devices are identical 


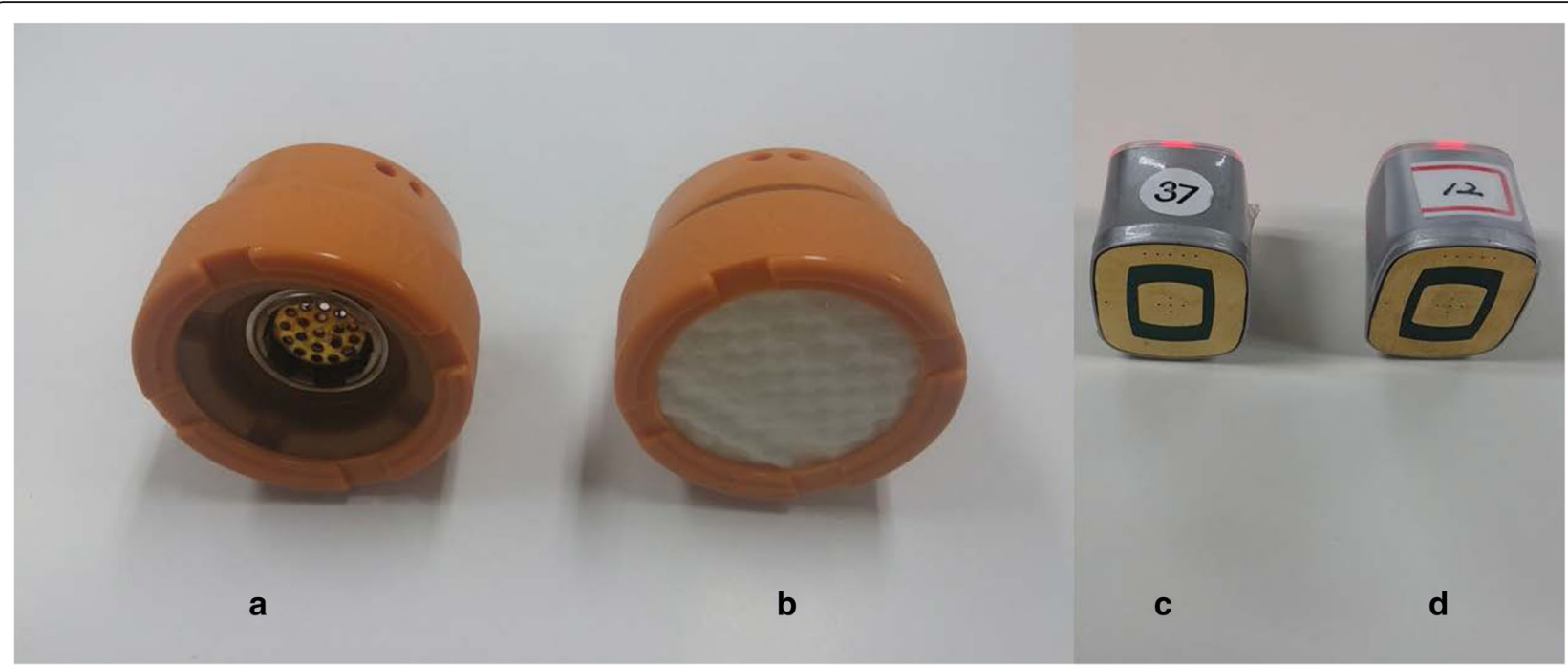

Fig. 6 A bottom view of moxibustion. $\mathbf{a} \& \mathbf{b}$, ignition-type moxibustion devices; $\mathbf{a}$, real; b, sham; c \& d: electrical moxibustion apparatus; c, real; d, sham

\section{Outcome measurements}

The following outcome measures will be assessed by assessors blinded to the group allocations.

\section{Primary outcome measure}

The mean change in average BFI scores from baseline to the end of the 8-week intervention will be the primary outcome measure of the study. The BFI is a questionnaire composed of 9 questions related to the degree of fatigue and the level of daily life interference caused by the fatigue [33]. Zero to ten points are assigned to each question ( $0=$ no fatigue or no interference; $10=$ the most severe level of fatigue or complete interference). The level of the fatigue can be grouped into three categories according to the average BFI score: 1-3 (mild), 4-6 (moderate), 7-10 (severe) [33].

\section{Secondary outcome measures}

The secondary outcome measures will include changes in average BFI scores from baseline to week 5 and week 13 and changes in the Functional Assessment of Cancer Therapy-Fatigue (FACT-F) scores, European Organization for Research and Treatment of Cancer Quality of Life Questionnaire C-30 (EORTC QLQ) scores, and Montreal Cognitive Assessment (MoCA) scores from the baseline to weeks 5,9 , and 13 .

\section{FACT-F}

The Functional Assessment of Cancer Therapy-General (FACT-G) is an assessment tool used to evaluate various functions in the daily lives of cancer patients [34]. It is comprised of 27 items in 4 domains: physical well-being, social/family well-being, emotional well-being, and functional well-being. The FACT-F is a combined questionnaire of the FACT-G plus a fatigue subscale including 13 items focused on fatigue and its related symptoms [34]. Using the FACT-F, diverse aspects of the life and the fatigue of cancer patients can be comprehensively assessed.

\section{EORTC QLQ C-30}

The EORTC QLQ C-30 was devised by the European Organization for Research and Treatment of Cancer (EORTC) to assess the QOL of cancer patients [35]. It includes 30 questions across the three domains of global health status/QOL, function scale and symptom scale. A variety of modules for specific cancer sites have been developed [35], although this study will adopt only the general version.

\section{MoCA}

It is known that many patients undergoing cancer treatment suffer from cognitive decline, and fatigue can affect the cognitive function of cancer patients [36]. The MoCA will be used in this study to evaluate the level of cognition of patients. This assessment tool was developed to screen for mild cognitive impairment, a transitional phase characterized by the cognitive changes that occur between early and normal dementia [37]. In this study, the MoCA will be used to detect patients with mild cognitive impairment and to assess changes in cognitive function.

\section{Safety outcomes}

The participants will be instructed to voluntarily report all AEs that occurred during the trial. The investigators 


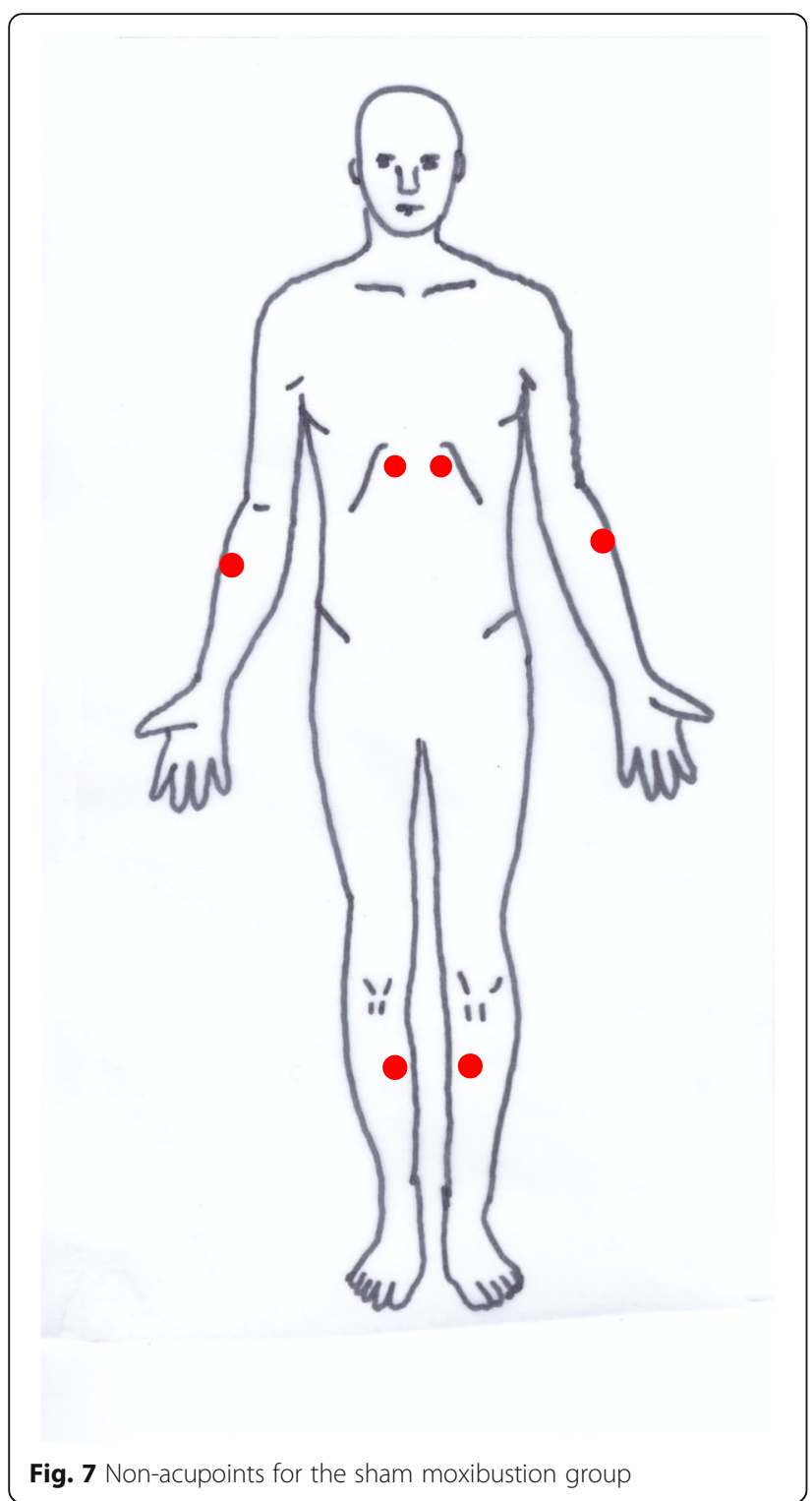

will determine whether the participants experienced any AEs via inquiries at each visit and checking for changes in laboratory tests from baseline to week 9. The laboratory tests will include complete blood count, differential count, absolute neutrophil count, aspartate aminotransferase, alanine aminotransferase, total bilirubin, gammaglutamyl transferase, blood urea nitrogen, creatinine, albumin, glucose, hemoglobin A1c, erythrocyte sedimentation rate, CRP, thyroid-stimulating hormone, free thyroxine, and human chorionic gonadotropin urine test (only for women in their childbearing years at screening visit). All AEs identified during the trial will be recorded in the clinical record form.

The severity of AEs will be classified into five grades according to the common terminology criteria for AEs
(CTCAE, version 4.0) developed by the National Cancer Institute as follows: mild, moderate, severe, lifethreatening, or death [38]. In cases for which the CTCAE is not applicable, Spilker's three level criteria will be used, and the $\mathrm{AE}$ will be classified into one of the three levels: mild, moderate, and severe [39].

A causal relationship between an $\mathrm{AE}$ and the moxibustion treatment provided in this study will be evaluated based on factors such as the temporal relationship, combined therapy, and underlying diseases, etc. The result of the causal relationship assessment will be categorized to one of the following six criteria: definitely related, probably related, possibly related, probably not related, definitely not related, or unknown.

\section{Blinding test and credibility test}

A blinding test will be conducted for the participants of the moxibustion group and the sham moxibustion group. Assessors who were not involved in the treatment procedure will administer the blind test questionnaire to the participants in these two groups after the first and last treatments of the trial. In the questionnaire, the participants will be asked to answer whether they think they had received real moxibustion, sham moxibustion, or that they had no idea. The result of this test will reveal whether patient blinding was successfully maintained in the trial.

Another questionnaire to evaluate the credibility of the intervention and the patient blinding will be provided to the participants of the two groups at the same time points. The credibility test is composed of four questions asking if the participant felt that the treatment could alleviate their fatigue, if they would recommend the treatment to a friend suffering from a similar complaint, if the treatment seems logical to them, and if the treatment could successfully alleviate other complaints. The result of the test will show the participants' expectations and confidence in the moxibustion treatment performed in this trial ranging from 0 (very low confidence) to 6 (very high confidence) [40].

\section{Data monitoring and quality control}

Data collection will be performed in accordance with the pre-approved protocols, and the quality of the study will be managed by regular monitoring. The monitoring will evaluate whether the recruitment and intervention procedures followed the protocol and that the record of the case report form matches the original document.

To ensure the quality of the study, all researchers should complete a good clinical practice training course. To maintain consistency of the procedures among research centers, we will provide detailed information of every step of this trial to all researchers at the researcher meetings and maintain communication 
Table 1 Schedule of enrolment, interventions, and assessments

\begin{tabular}{|c|c|c|c|c|c|c|c|}
\hline & \multicolumn{7}{|c|}{ STUDY PERIOD } \\
\hline & \multirow{2}{*}{$\begin{array}{c}\text { Enrolment } \\
-w k 1\end{array}$} & \multirow{2}{*}{$\begin{array}{c}\text { Allocation } \\
\text { wko }\end{array}$} & \multicolumn{4}{|c|}{ Post-allocation } & \multirow{2}{*}{$\begin{array}{c}\text { Close-out } \\
w k_{13}\end{array}$} \\
\hline TIMEPOINT & & & $w_{k}$ & $w_{5}$ & wk8 & wkg & \\
\hline \multicolumn{8}{|l|}{ ENROLMENT: } \\
\hline \multirow{3}{*}{$\begin{array}{r}\text { Eligibility screen } \\
\text { Informed consent } \\
\text { Allocation }\end{array}$} & $\mathrm{X}$ & & & & & & \\
\hline & $\mathrm{X}$ & & & & & & \\
\hline & & $\mathrm{X}$ & & & & & \\
\hline \multicolumn{8}{|l|}{ INTERVENTIONS: } \\
\hline \multicolumn{8}{|l|}{ Moxibustion } \\
\hline \multicolumn{8}{|l|}{ Sham moxibustion } \\
\hline \multicolumn{8}{|l|}{ Usual care } \\
\hline \multicolumn{8}{|l|}{ ASSESSMENTS: } \\
\hline$B F I$ & $\mathrm{X}$ & $\mathrm{X}$ & & $X$ & & $\mathrm{X}$ & $X$ \\
\hline$F A C T-F$ & & $\mathrm{X}$ & & $\mathrm{X}$ & & $\mathrm{X}$ & $\mathrm{X}$ \\
\hline EORTC QLQ-C30 & & $\mathrm{X}$ & & & & $\mathrm{X}$ & $\mathrm{X}$ \\
\hline MoCA & & $\mathrm{X}$ & & & & $\mathrm{X}$ & \\
\hline CHDE & & $\mathrm{X}$ & & & & & \\
\hline Blinding test ${ }^{\mathrm{a}}$ & & & $\mathrm{X}$ & & $\mathrm{X}$ & & \\
\hline Credibility test $^{\mathrm{a}}$ & & & $\mathrm{X}$ & & $\mathrm{X}$ & & \\
\hline Adverse event & & & $\mathrm{X}$ & $\mathrm{X}$ & $\mathrm{X}$ & $\mathrm{X}$ & $\mathrm{X}$ \\
\hline Laboratory test $^{b}$ & $\mathrm{X}$ & & & & & $\mathrm{X}$ & \\
\hline
\end{tabular}

BFI Brief Fatigue Inventory, FACT-F Functional Assessment of Cancer Therapy-Fatigue, EORTC QLQ-C30 European Organization for Research and Treatment of Cancer Quality of Life Questionnaire, MoCA Montreal Cognitive Assessment, CHDE Questionnaire for Cold-Heat \& Deficiency-Excess Patternization ${ }^{a}$ The blinding and credibility tests will be performed only for the moxibustion and sham moxibustion groups

${ }^{\mathrm{b}}$ The laboratory test includes complete blood count and differential count, absolute neutrophil count, aspartate aminotransferase, alanine aminotransferase, total bilirubin, gamma-glutamyl transferase, blood urea nitrogen, creatinine, albumin, glucose, hemoglobin A1c, erythrocyte sedimentation rate, C-reactive protein, thyroid-stimulating hormone, free thyroxine, and human chorionic gonadotropin urine test (only for women in their childbearing years at the screening visit)

with all researchers throughout the study period. The practitioners will be limited to those who have been licensed as a doctor of Korean medicine by the Ministry of Health and Welfare of South Korea and have a clinical career of 2 or more years.

\section{Sample size calculation}

The primary outcome of this study will be the mean change in average BFI scores from baseline to week 9 . Based on a previous study [41], we estimated that the mean difference of the pre- and post-treatment change in the average BFI scores between the moxibustion and sham moxibustion group will be -1.6 and the standard deviation will be 2.0 . With a $5 \%$ significance level and $80 \%$ power, the sample size was calculated to be 25 .
Considering a $20 \%$ drop-out rate, 32 participants are required per group. In conclusion, a total of 96 participants are needed in this trial.

\section{Statistical analysis}

An independent statistician blinded to group allocation will perform statistical analysis using $\mathrm{SAS}^{\circ}$ Version 9.4 (SAS institute. Inc., Cary, NC). All statistical analyses will be two-tailed, and the significance level will be set at $5 \%$.

Three types of analysis sets will be used in this study, as follows: intention to treat (ITT) analysis set, full analysis set (FAS), and per-protocol (PP) set. The ITT analysis set will be comprised of the data from every participant who had been randomly assigned to any 
group in this trial. The FAS set will include the data from all participants who ever received the intervention and provided assessment data at least once following random allocation. The PP set will include only the data from participants who completed the trial without any major violation of the protocol. The FAS will be the primary set for the efficacy analysis, and sensitivity analysis will be conducted to compare the results from the FAS and PP sets. For safety analysis, the ITT set will be the primary set, and the PP set will be used as a supplement. The multiple imputation method will be adopted to handle missing data in incomplete data sets.

Summary statistics of demographic characteristics and baseline measurements of the variables of each group will be presented. Continuous data will be expressed as the mean \pm standard deviation, but median and 95\% confidence intervals will be provided if necessary. Between-group differences will be analyzed using analysis of variance (ANOVA) or the Kruskal-Wallis test. Categorical data will be presented as frequencies with percentiles and analyzed using the Chi-square test or the Fisher's exact test.

The primary outcome measure of this study will be the mean change of average BFI scores acquired at baseline and week 9. To validate significant changes in BFI between groups, we assumed two sets of hypotheses.

The null hypothesis $\left(\mathrm{H}_{01}\right)$ : There is no difference in the mean change of the BFI before and after treatment between the moxibustion group and the usual care group.

The alternative hypothesis $\left(\mathbf{H}_{11}\right)$ : There is a difference in the mean change of the BFI before and after treatment between the moxibustion group and the usual care group.

The null hypothesis $\left(\mathrm{H}_{02}\right)$ : There is no difference in the mean change of the BFI before and after treatment between the moxibustion group and the sham moxibustion group.

The alternative hypothesis $\left(\mathrm{H}_{12}\right)$ : There is a difference in the mean change of the BFI before and after treatment between the moxibustion group and the sham moxibustion group.

To test the two hypotheses, analysis of covariance (ANCOVA) will be utilized with the baseline BFI value as a covariate and each group as a fixed factor. If any clinically significant difference between groups is identified among the demographic characteristics or the variables measured at baseline, it will be adjusted based on its covariance. The problem of multiple comparison will be solved using the fixed sequence method. In other words, if the $\mathrm{H}_{01}$ is rejected and $\mathrm{H}_{11}$ is adopted in the statistical test for the first hypothesis set, and then the test for $\mathrm{H}_{02}$ will performed. However, when the $\mathrm{H}_{01}$ is adoptted, the second hypothesis set for $\mathrm{H}_{02}$ will not be verified [42].
The secondary outcome measures of the study will be the mean changes in the BFI, FACT-F, EORTC QLQ C30 and MoCA before and after the interventions in the three groups. They will be analyzed via the same methods used for the primary outcome measure analysis. Student's paired t-tests or Wilcoxon signed-rank tests will be used to analyze the differences in the measured values before and after treatment in each group. ANOVA with Dunnett's post hoc test will be performed to compare the differences in trends per visit. Subgroup analyses will be conducted to check whether there are significant differences in the responses to moxibustion treatment depending on the severity of fatigue, the expected level of treatment, or patterns of cold-heat and deficiency-excess measured at baseline.

\section{Discussion}

The purpose of this study is to assess the efficacy and safety of moxibustion treatment for patients with cancer-related fatigue.

Cancer-related fatigue is one of the most prominent manifestations of cancer survivors that can be occur over a long period of time even after the end of cancer treatment or the complete remission of cancer [3]. Although the prevalence of cancer-related fatigue varies among studies, it is generally reported that approximately 60 to $100 \%$ of cancer survivors experience cancer-related fatigue [43]. Cancer-related fatigue lowers the QOL of the family of the patient in addition to the cancer patient and can become a socially obvious burden [5-7]. Although a variety of pharmacologic and nonpharmacologic therapies for treating cancer-related fatigue have been evaluated in the clinical trials, no treatments have been confirmed as valid and safe for long-term use $[17,19]$. Therefore, it is important to confirm whether moxibustion, one of the oldest but most popular therapies in traditional East Asian medicine, including Korean medicine, is a viable therapeutic option for the management of cancer-related fatigue.

The moxibustion treatment regimen was established based on the findings of the relative studies that were previously published. Although there were few studies with good quality evaluating the effect of moxibustion for the patients with cancer-related fatigue, a recent publication by Mao et al. [32]. using infrared laser moxibustion was helpful. Mao et al. [32]. reported that the moxibustion reduced the BFI score during the 4-week treatment period, however, it tended to stagnate at follow-up session at week 8 . Another study by Mao et al. [44] targeting the breast cancer patients with psychological distress including cancer-related fatigue was also reliable even though its intervention was electroacupuncture, not moxibustion. According to this study, the BFI score decreased during the 8-week of treatment 
period, and then the improvement trend was stopped 4 weeks after the completion of the treatment. Therefore, we expect to see how the BFI will change during and after the 8-week treatment period.

It is known that the placebo effect has a high proportion in the palliative care for the cancer survivors with cancer-related fatigue [45]. It varies by $27-60 \%$ depending on the studies [45]. Besides, the sham control interventions of traditional medicine such as moxibustion or acupuncture are not reported to be physiologically inert [46, 47]. Considering the clinical characteristics of the cancer-related fatigue which is sensitive to the placebo response and the unique nature of the sham moxibustion, we cannot rule out the possibility that the difference between the effects of real moxibustion and sham moxibustion will not be statistically significant even though the moxibustion treatment is actually effective because of the placebo effect. This is why we designed a 3 -arm parallel study of the usual care group as well as the sham control group.

There are some limitations to this study. First, the outcome measures do not include objective indicators for assessing the level of fatigue. Because fatigue itself is defined as the subjective feeling of the patient, there is currently no way to objectively evaluate it. Some studies have reported that various biomarkers, such as TNFalpha, interleukin (IL)-1 beta, IL-6, IL-2, CRP, etc., are associated with cancer-related fatigue $[6,10]$, although the results of these studies are not consistent in regarding this issue. Therefore, there is currently no confirmed biomarker that represents the level of cancer-related fatigue [48]. When definitive biomarkers are discovered in the future, additional clinical trials will need to consider them. Nevertheless, the use of questionnaires to regarding the level of the subjective feelings of fatigue of the subjects is the most widely used method to assess or quantify fatigue as objectively as possible in the field, and we adopted the BFI and FACT-F according to this gold-standard.

Second, there is a limitation pertaining to incomplete blinding. We used moxibustion devices that appeared to be identical in the moxibustion and sham moxibustion groups in an attempt to achieve patient blinding between these two groups. However, it is not possible to blind the participants in the usual care group that do not receive moxibustion treatment. Thus, there could be a concern that this incomplete blinding of participants in the usual care group may introduce bias into the results regarding the outcome measures based on the subjective symptoms that the patients feel.

However, Atkinson et al. [49] recently showed that there is no clear evidence that the patient blinding is a source of bias in the patient-reported outcome (PRO) or at least that the bias significantly affects the results of the PRO. They insisted that, in clinical trials of therapies for cancer patients, the use of PRO, which is essential for directly assessing the risks, benefits, and value of the therapies to cancer patients, should be promoted regardless of patient blinding because there is no other means that provides information by completely capturing the patient's experience related to the treatment [49]. Based on such expert opinions, we will adopt a three-armed parallel design, including a usual care group in addition to a sham control group, in this trial even though the primary outcome measure is highly dependent on the patients' subjectivity.

There is another limitation in terms of patient blinding. The moxibustion delivered to the sham group is designed to not deliver complete warmth to the site of application; therefore, the patients of this group may notice that the treatment is not real moxibustion because it is not sufficiently hot. To prevent this possibility, only patients who have never received moxibustion treatment should be recruited for inclusion. However, this inclusion criterion could significantly lower the recruitment rate. Thus, we decided to register patients regardless of their past experience using moxibustion treatment. To further mitigate this limitation, the real and sham moxibustion devices used in the study will have the same appearance. In addition, the patients will be allowed observe the procedures that the practitioner carries out while operating the moxibustion devices: In case of IM, the participants of both groups will see the smoke and smell the odor from the burning mugwort moxa cone after the practitioner ignites it; and in case of EM, the participants will see the practitioner turn on the EM, which will begin to blink. Finally, we will perform a blinding test to confirm whether patient blinding had been successfully achieved in these two groups.

There are a variety of types in cancer, although patients with cancer-related fatigue will be allowed to participate in this study regardless of cancer type. Some researchers have suggested that patients with cancer at specific sites may be more vulnerable to cancer-related fatigue [14], although it has not been clearly investigated how specific types of cancer develop or affect fatigue. Therefore, we will comprehensively recruit patients regardless of cancer type in this study. However, if the results of this study or future studies by experts in the field demonstrate more promising clues regarding the relationship between specific cancer types and cancerrelated fatigue, a new clinical trial targeting only patients with limited types of cancer should be designed.

Cancer-related fatigue is a symptom that patients can experience before cancer diagnosis, during cancer progression, during cancer treatment, after cancer treatment, or even after cancer remission. However, 'during cancer treatment' and 'immediately after cancer treatment' are 
known time periods during which cancer-related fatigue is most common and most prominent [6]. It has also been reported that the type of cancer treatment such as chemotherapy or radiotherapy can contribute in different ways to cancer-related fatigue [50]. The regimen of the treatment, for example, the type of drugs or the dose of radiation, might differentially affect fatigue. However, considering the practical feasibility of the clinical trial, we decided to exclude those who are currently undergoing active treatment for cancer or scheduled to receive cancer treatment in the near future. Therefore, the results of this study will not shed light on the effect of moxibustion at the peak of cancer-related fatigue.

One of the distinct features of this study is that the participants will be categorized based on patterns as cold or hot and as deficiency or excess. In traditional medicine, pattern identification is a way to categorize a patient according to the signs and symptoms of several patterns, and doctors can determine how to treat the patient based on the identified patterns. Moxibustion is a widely used intervention in traditional Korean medicine and is often chosen as a better option, especially for patients categorized by cold or deficiency patterns. For this reason, we will adopt the questionnaire for cold-hot and deficiency-excess pattern categorization [51]. We will conduct subgroup analyses according to the identified patterns to determine whether moxibustion is more effective in cold-type patients than in hot-type patients and whether it improves the symptoms of deficiencytype patients better than excess-type patients. We will also determine whether such patterns are similar or different between the sham moxibustion group and the usual care group. We believe that such an analysis is a meaningful scientific exploration for investigating the clinical and practical value of the concept in traditional Korean medical theory, and the results of this study will help to identify patients who are best suited for moxibustion and related therapies.

Another characteristic of this study is that it assesses not only fatigue but other biobehavioral factors, such as QOL and cognitive function. A previous study reported that cancer-related fatigue negatively influences the QOL and cognitive function of patients [52]. Thus, we seek to comprehensively evaluate the effect of moxibustion on various aspects of daily life that patients with cancer-related fatigue experience.

A variety of factors are known to influence the cancerrelated fatigue as well as cancer treatment and cancer itself [11]. In this study, the patients with prominent risk factors that are known to contribute to cancer-related fatigue will be screened out to eliminate confounding factors and to focus on cancer-related fatigue. Thus, the eligibility criteria include the exclusion of patients who have current severe inflammation or anemia, clinically significant insomnia, moderate or higher levels of emotional disturbances such as anxiety or depression, and definite cancer pain. The attempt to select purely tired cancer patients with minimal other pathological causes of fatigue is a strength of this study, although as a result, the results of this study cannot be applied to patients with cancer-related fatigue combined with these conditions. Therefore, these results as they apply to patients with multiple conditions need to be further examined in future studies.

\section{Trial status}

The trial is currently recruiting participants.

\section{Additional files}

Additional file 1: Details of moxibustion and sham moxibustion treatments based on the Standards for Reporting Interventions in Clinical Trials of Acupuncture (STRICTA) Checklist. (DOCX 20 kb)

Additional file 2: Standard Protocol Items: Recommendations for Interventional Trials (SPIRIT) checklist. (DOC $123 \mathrm{~kb}$ )

\section{Abbreviations}

AE: Adverse event; ANCOVA: Analysis of covariance; ANOVA: Analysis of variance; $\mathrm{BFI}$ : Brief Fatique Inventory; CAM: Complementary alternative medicine; CONSORT: Consolidated Standards of Reporting Trials; CRP: Creactive protein; CTCAE: Common terminology criteria for adverse events; ECOG: The Eastern Cooperative Oncology Group; EM: Electrical moxibustion apparatus; EORTC QLQ C-30: European Organization for Research and Treatment of Cancer Quality of Life Questionnaire; EORTC: European Organization for Research and Treatment of Cancer; FACT-F: Functional Assessment of Cancer Therapy-Fatigue; FACT-G: Functional Assessment of Cancer Therapy-General; HADS: Hospital Anxiety and Depression Scale; HPA: Hypothalamic-pituitary-adrenal; ICD-10-CM: International Classification of Diseases, tenth revision clinical modification; IL: Interleukin; IM: Ignitiontype moxibustion device; ISI: Insomnia severity index; MoCA: Montreal Cognitive Assessment; NRS: Numeric rating scale; PRO: Patient-reported outcome; QOL: Quality of life; SAE: Serious adverse reaction;

STRICTA: Standards for Reporting Interventions in Clinical Trials of Acupuncture; TNF: Tumor necrosis factor

\section{Acknowledgements}

We gratefully acknowledge all the collaborators and participants of the study. We especially appreciate professor Sookyung Lee for helping us to reflect the clinical characteristics of cancer patients in the study protocol.

\section{Funding}

This study was financially supported by funds from the Korea Institute of Oriental Medicine (K16122 \& K17122).

\section{Availability of data and materials}

It is a protocol of ongoing clinical trial and therefore there is no finding to report yet. After completion of this trial, data requested for public purposes will be provided.

\section{Authors' contributions}

MK designed the study and drafted the manuscript. JEK, HYL, ARK, and HJP participated in the design of the study. OJK designed the plan for the statistical analysis of the study. EJK, YCP, BKS and JHC provided technical advice and made critical revisions. JHK participated in the design of the study as a principal investigator and has the final responsibility for the decision to submit this manuscript for publication. All authors read and approved the final manuscript. 


\section{Ethics approval and consent to participate}

The study protocol has been prepared according to the Standard Protocol Items: Recommendations for Intervention Trials (see Additional file 2) and approved by the institutional review boards of the hospitals to which the participating research centers belong: Daejeon Oriental Hospital of Daejeon University (djomc-141-1), Kyung Hee University Oriental Hospital at Gangdong (KHNMCOH 2016-09-004), and Dongguk University Bundang Oriental Hospital (2016-0008).

The study will be performed in accordance with the approved protocol, and written consent will be obtained from every participant.

This trial is registered with the Clinical Research Information Service of South Korea (CRIS-KCT0002170).

\section{Consent for publication}

Not applicable.

\section{Competing interests}

The authors declare that they have no competing interests.

\section{Publisher's Note}

Springer Nature remains neutral with regard to jurisdictional claims in published maps and institutional affiliations.

\section{Author details \\ ${ }^{1}$ Clinical Research Division, Korea Institute of Oriental Medicine, 1672, Yuseongdae-ro, Yuseong-gu, Daejeon 34054, Republic of Korea. ${ }^{2}$ College of Korean Medicine, Dongguk University, 123, Dongdae-ro, Gyeongju, Gyeongsangbuk-do 38066, Republic of Korea. ${ }^{3}$ Department of Acupuncture \& Moxibustion, Kyung Hee University Hospital at Gangdong, 892, Dongnam-ro, Gangdong-gu, Seoul 05278, Republic of Korea. ${ }^{4}$ Department of Internal Medicine, College of Traditional Korean Medicine, Daejeon University, 62, Daehak-ro, Dong-gu, Daejeon 34520, Republic of Korea.}

Received: 12 January 2017 Accepted: 22 June 2017

\section{Published online: 05 July 2017}

\section{References}

1. American Cancer Society. Cancer Facts \& Figures 2015. Atlanta: American Cancer Society; 2015. http://www.cancer.org/acs/groups/content/@editorial/ documents/document/acspc-044552.pdf. Accessed 5 Jun 2017.

2. Bower JE. Prevalence and causes of fatigue after cancer treatment: the next generation of research. J Clin Oncol. 2005;23(33):8280-2.

3. Garland SN, Roscoe JA, Heckler CE, Barilla H, Gehrman P, Findley JC, et al, Effects of armodafinil and cognitive behavior therapy for insomnia on sleep continuity and daytime sleepiness in cancer survivors. Sleep Med. 2016;20:18-24

4. Berger AM, Gerber LH, Mayer DK. Cancer-related fatigue. Cancer. 2012:118(S8):2261-9.

5. Groenvold M, Petersen MA, Idler E, Bjorner JB, Fayers PM, Mouridsen HT. Psychological distress and fatigue predicted recurrence and survival in primary breast cancer patients. Breast Cancer Res Treat. 2007;105(2):209-19.

6. Bower JE, Ganz PA, Irwin MR, Kwan L, Breen EC, Cole SW. Inflammation and behavioral symptoms after breast cancer treatment: do fatigue, depression, and sleep disturbance share a common underlying mechanism? J Clin Oncol. 2011;29(26):3517-22.

7. Gupta D, Lis CG, Grutsch JF. The relationship between cancer-related fatigue and patient satisfaction with quality of life in cancer. J Pain Symptom Manag. 2007;34(1):40-7.

8. Stasi R, Abriani L, Beccaglia P, Terzoli E, Amadori S. Cancer-related fatigue: evolving concepts in evaluation and treatment. Cancer. 2003;98(9):1786-801.

9. Lee B-N, Dantzer R, Langley KE, Bennett GJ, Dougherty PM, Dunn AJ, et al. A cytokine-based neuroimmunologic mechanism of cancer-related symptoms. Neuroimmunomodulation. 2004;11(5):279-92.

10. Bower JE. Cancer-related fatigue-mechanisms, risk factors, and treatments. Nat Rev Clin Oncol. 2014;11(10):597-609.

11. Ryan JL, Carroll JK, Ryan EP, Mustian KM, Fiscella K, Morrow GR. Mechanisms of cancer-related fatigue. Oncologist. 2007;12(Suppl 1):22-34.

12. Nicolson GL, Settineri R. Lipid replacement therapy: a functional food approach with new formulations for reducing cellular oxidative damage, cancer-associated fatigue and the adverse effects of cancer therapy. Funct Foods Health Dis. 2011;1(4):135-60.
13. Jacobsen PB, Donovan KA, Small BJ, Jim HS, Munster PN, Andrykowski MA Fatigue after treatment for early stage breast cancer. Cancer. 2007;110(8):1851-9.

14. Gerber LH. Cancer-related fatigue: persistent, pervasive, and problematic. Phys Med Rehabil Clin N Am. 2017;28(1):65-88.

15. Tian L, Lin L, Li HL, Chen KJ, Zhang XJ, Qian SJ, et al. Prevalence and associated factors of cancer-related fatigue among cancer patients in eastern China. Oncologist. 2016;21:1349-54.

16. Berger AM, Mooney K, Alvarez-Perez A, Breitbart WS, Carpenter KM, Cella D, et al. Cancer-related fatigue, version 2.2015. J Natl Compr Cancer Netw. 2015;13(8):1012-39.

17. Qu D, Zhang Z, Yu X, Zhao J, Qiu F, Huang J. Psychotropic drugs for the management of cancer-related fatigue: a systematic review and metaanalysis. Eur J Cancer Care. 2015;25(6):970-9.

18. Mücke M, Cuhls H, Peuckmann-Post V, Minton O, Stone P, Radbruch L. Pharmacological treatments for fatigue associated with palliative care. Cochrane Database Syst Rev. 2015;5:CD006788.

19. Lau CH, Wu X, Chung VC, Liu X, Hui EP, Cramer H, et al. Acupuncture and related therapies for symptom management in palliative cancer care: systematic review and meta-analysis. Medicine. 2016;95(9):e2901.

20. Sood A, Barton DL, Bauer BA, Loprinzi CL. A critical review of complementary therapies for cancer-related fatigue. Integr Cancer Ther. 2007;6(1):8-13.

21. Davis EL, Oh B, Butow PN, Mullan BA, Clarke S. Cancer patient disclosure and patient-doctor communication of complementary and alternative medicine use: a systematic review. Oncologist. 2012;17(11):1475-81.

22. Rush CL, Lobo T, Serrano A, Blasini M, Campos C, Graves KD: Complementary and alternative medicine use and latina breast cancer survivors' symptoms and functioning. Healthcare 2016, 31;4(4):80.

23. Kim S-G, Park E-C, Park J-H, Hahm M-I, Lim J-H, Choi K-S. Initiation and discontinuation of complementary therapy among cancer patients. J Clin Oncol. 2007;25(33):5267-74.

24. Korean Acupuncture \& Moxibustion Society Textbook Compilation Committee. The acupuncture and moxibustion. Seoul, Jipmoondang; 2008. p. 393-5.

25. Deng $H$, Shen $X$. The mechanism of moxibustion: ancient theory and modern research. Evid Based Complement Alternat Med. 2013;2013:379291.

26. Kim HG, Yoo SR, Park HJ, Son C-G. Indirect moxibustion (CV4 and CV8) ameliorates chronic fatigue: a randomized, double-blind, controlled study. J Altern Complement Med. 2013;19(2):134-40.

27. Shu Q, Wang H, Litscher D, Wu S, Chen L, Gaischek I, et al. Acupuncture and moxibustion have different effects on fatigue by regulating the autonomic nervous system: a pilot controlled clinical trial. Sci Rep. 2016;6:37846.

28. Yi T, Qi L, Li J, Le J-J, Shao L, Du X, et al. Moxibustion upregulates hippocampal progranulin expression. Neural Regen Res. 2016;11(4):610-6.

29. Liu J, Zhao B, Cui Y, Huang Y, Huang C, Huang J, et al. Effects of shenque moxibustion on behavioral changes and brain oxidative state in apolipoprotein E-deficient mice. Evid Based Complement Alternat Med. 2015;2015:804804

30. Kogure M, Mimura N, Ikemoto H, Ishikawa S, Nakanishi-Ueda T, Sunagawa $M$, et al. Moxibustion at mingmen reduces inflammation and decreases IL-6 in a collagen-induced arthritis mouse model. J Acupunct Meridian Stud. 2012;5(1):29-33.

31. Lee S, Jerng UM, Liu Y, Kang JW, Nam D, Lee J-D. The effectiveness and safety of moxibustion for treating cancer-related fatigue: a systematic review and meta-analyses. Support Care Cancer. 2014;22(5):1429-40.

32. Mao H, Mao JJ, Guo M, Cheng K, Wei J, Shen X, et al. Effects of infrared laser moxibustion on cancer-related fatigue: a randomized, double-blind, placebo-controlled trial. Cancer. 2016;122(23):3667-72.

33. Mendoza TR, Wang XS, Cleeland CS, Morrissey M, Johnson BA, Wendt JK, et al. The rapid assessment of fatigue severity in cancer patients. Cancer. 1999; 85(5):1186-96.

34. Yellen SB, Cella DF, Webster K, Blendowski C, Kaplan E. Measuring fatigue and other anemia-related symptoms with the functional assessment of cancer therapy (FACT) measurement system. J Pain Symptom Manag. 1997;13(2):63-74.

35. Aaronson NK, Ahmedzai S, Bergman B, Bullinger M, Cull A, Duez NJ, et al. The European Organization for Research and Treatment of cancer QLQ-C30: a quality-of-life instrument for use in international clinical trials in oncology. J Natl Cancer Inst. 1993;85(5):365-76. 
36. Curt GA, Breitbart W, Cella D, Groopman JE, Horning SJ, Itri LM, et al. Impact of cancer-related fatigue on the lives of patients: new findings from the fatigue coalition. Oncologist. 2000;5(5):353-60.

37. Nasreddine ZS, Phillips NA, Bédirian V, Charbonneau S, Whitehead V, Collin I, et al. The Montreal cognitive assessment, MoCA: a brief screening tool for mild cognitive impairment. J Am Geriatr Soc. 2005;53(4):695-9.

38. U.S. Department of health and human services. Common terminology criteria for adverse events (CTCAE), version 4.0. 2009. https://evs.nci.nih.gov/ ftp1/CTCAE/CTCAE_4.03_2010-06-14_QuickReference_5x7.pdf. Accessed 5 June 2017.

39. Spilker B. Quality of life and pharmacoeconomics in clinical trials. 2nd ed. Philadelphia: Lippencott-Raven; 1995.

40. Devilly GJ, Borkovec TD. Psychometric properties of the credibility/ expectancy questionnaire. J Behav Ther Exp Psychiatry. 2000;31(2):73-86.

41. Smith C, Carmady B, Thornton C, Perz J, Ussher JM. The effect of acupuncture on post-cancer fatigue and well-being for women recovering from breast cancer: a pilot randomised controlled trial. Acupunct Med. 2013:31(1):9-15

42. Wiens BL. A fixed sequence Bonferroni procedure for testing multiple endpoints. Pharm Stat. 2003;2(3):211-5.

43. Weis J. Cancer-related fatigue: prevalence, assessment and treatment strategies. Expert Rev Pharmacoecon Outcomes Res. 2011;11(4):441-6.

44. Mao JJ, Farrar JT, Bruner D, Zee J, Bowman M, Seluzicki C, et al. Electroacupuncture for fatique, sleep, and psychological distress in breast cancer patients with aromatase inhibitor-related arthralgia: a randomized trial. Cancer. 2014;120(23):3744-51.

45. Yennu S, Lim K-H, Williams JL, Lu Z, Bruera E. Frequency and factors associated with placebo response in cancer-related fatigue treatment trials. J Clin Oncol. 2016;34(26_suppl):210.

46. Ren X, Yao C, Wu F, Li Z, Xing J, Zhang H. Effectiveness of Moxibustion treatment in quality of life in patients with knee osteoarthritis: a randomized, double-blinded, placebo-controlled trial. Evid Based Complement Alternat Med. 2015;2015:569523.

47. Linde K, Niemann K, Schneider A, Meissner K. How large are the nonspecific effects of acupuncture? A meta-analysis of randomized controlled trials. BMC Med. 2010;8(1):75.

48. Carlson LE, Campbell TS, Garland SN, Grossman P. Associations among salivary cortisol, melatonin, catecholamines, sleep quality and stress in women with breast cancer and healthy controls. J Behav Med. 2007;30(1):45-58.

49. Atkinson TM, Wagner J-S, Basch E. Trustworthiness of patient-reported outcomes in unblinded cancer clinical trials. JAMA Oncol. 2016; doi:10.1001/ jamaoncol.2016.3328.

50. Eyob T, Ng T, Chan R, Chan A. Impact of chemotherapy on cancer-related fatigue and cytokines in 1312 patients: a systematic review of quantitative studies. Curr Opin Support Palliat Care. 2016;10(2):165-79.

51. Kwon OS, Cho SJ, Choi K-H, Yeon SH, Kang S-Y, Kim J-H, et al. Safety recommendations for moxa use based on the concentration of noxious substances produced during commercial indirect moxibustion. Acupunct Med. 2017;35(2):93-9.

52. Hofman $M$, Ryan JL, Figueroa-Moseley CD, Jean-Pierre P, Morrow GR. Cancer-related fatigue: the scale of the problem. Oncologist. 2007;12(Supplement 1):4-10.

\section{Submit your next manuscript to BioMed Central and we will help you at every step:}

- We accept pre-submission inquiries

- Our selector tool helps you to find the most relevant journal

- We provide round the clock customer support

- Convenient online submission

- Thorough peer review

- Inclusion in PubMed and all major indexing services

- Maximum visibility for your research

Submit your manuscript at www.biomedcentral.com/submit
Biomed Central 